\title{
Reverse bilateral latissimus dorsi flap reconstruction after extensive mid back dermatofibrosarcoma protuberans excision: a case report
}

\author{
Stefano Bonomi ${ }^{1}$, Laura Sala ${ }^{1}$, Alessandro Gronchi², Dario Callegaro ${ }^{2}$, Umberto Cortinovis ${ }^{1}$ \\ 'Plastic and Reconstructive Surgery Unit, Fondazione IRCCS Istituto Nazionale dei Tumori, Via Venezian 1, Milan 20133, Italy. \\ ${ }^{2}$ Sarcoma Surgery Unit, Fondazione IRCCS Istituto Nazionale dei Tumori, Via Venezian 1, Milan 20133, Italy.
}

Correspondence to: Dr. Stefano Bonomi, Plastic and Reconstructive Surgery Unit, Fondazione IRCCS Istituto Nazionale dei Tumori, Via Venezian 1, Milan 20133, Italy. E-mail: stefano.bonomi@istitutotumori.mi.it

How to cite this article: Bonomi S, Sala L, Gronchi A, Callegaro D, Cortinovis U. Reverse bilateral latissimus dorsi flap reconstruction after extensive mid back dermatofibrosarcoma protuberans excision: a case report. Plast Aesthet Res 2018;5:8. http://dx.doi.org/10.20517/2347-9264.2017.93

Received: 20 Dec 2017 First Decision: 23 Jan 2018 Revised: 2 Feb 2018 Accepted: 9 Feb 2018 Published: 6 Mar 2018

Science Editor: Taina A. Partanen Copy Editor: Jun-Yao Li Production Editor: Cai-Hong Wang

\begin{abstract}
Surgical resection of soft tissue sarcoma of the trunk can result in large defects requiring complex reconstruction for coverage of vital neurovascular structures and tissue defect. Large defects of the back could be reconstructed with multiple random pattern or local pedicled flaps. We present the case of a 48 -year-old patient with a locally advanced dermatofibrosarcoma protuberans of the back. Wide local excision of the lesion was performed. The soft tissue defect measured $22 \mathrm{~cm} \times 20 \mathrm{~cm} \times 4 \mathrm{~cm}$ and was reconstructed with bilateral reverse latissimus dorsi myocutaneous (RLDM) flap. Each RLDM flap measured $24 \mathrm{~cm} \times 10 \mathrm{~cm}$. The donor site on the back was closed directly on both sides. The patient recovered well and the two flaps healed uneventfully. Twelve months after surgery the patient is disease-free. The use of a RLDM flap in mid-back reconstructions provided wide well-vascularized soft tissue, minimized risk of infection, and maximized back coverage. This flap is an excellent choice for reconstruction of large defects of the mid-back.
\end{abstract}

Keywords: Reverse latissimus dorsi myocutaneous flap, trunk reconstruction, posterior trunk defect, sarcoma, dermatofibrosarcoma protuberans

\section{INTRODUCTION}

Soft tissue sarcomas (STS) represent less than $1 \%$ of all malignant tumors. STS can occur anywhere in the body but $75 \%$ are located in the extremities, $10 \%$ in the trunk and $10 \%$ in the retroperitoneum ${ }^{[1]}$. The mainstay

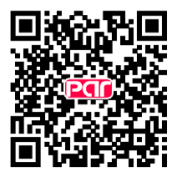


of treatment of localized STS is the complete surgical resection of the tumor with adequate margins. Both the width and quality of the surgical margins are crucial in determining patients prognosis. Low-risk tumors are typically managed with a wide surgical resection alone. Patients with high-risk tumors may be treated with adjuvant/neoadjuvant radiotherapy and/or chemotherapy to minimize respectively the local and systemic risk of failure.

Dermatofibrosarcoma protuberans (DFSP) is a low-grade, locally aggressive, STS of the cutis. Typically, DFSP arises within the dermis as a slow-growing plaque or nodule and subsequently spread to the subcutaneous tissues. The pathogenetic driver of this tumor is the $\mathrm{t}(17 ; 22)(\mathrm{q} 22 ; \mathrm{q} 13)$ translocation which leads to the formation of COL1A1-platelet-derived growth factor (PDGF) beta fusion transcripts. The overproduction of the PDGF beta-chain stimulates tumor cells growth with an autocrine loop ${ }^{[2]}$.

DFSP are managed with wide excision of the tumor en-bloc with surrounding soft tissues. The Mohs micrographic surgery is an option to minimize the amount of tissue resected, especially in critical areas ${ }^{[3]}$. The risk of local recurrence is in the range of $1 \%-4 \%$ at 10 years after wide surgery and the metastatic risk is about $2 \%$ at 10 years in major series ${ }^{[4,5]}$. The risk of local failure increases significantly in case of marginal or microscopically positive resection, thus the quality of surgery is critical. In a minority of patients, the tumor may harbour a fibrosarcomatous transformation which is associated with a much higher systemic risk. The most common site of DFSP occurrence is the trunk (72\%). Here, the extensive removal of tissue often requires reconstructive surgery because primary closure is not possible. Furthermore, in case of local recurrences, the re-resections of superficial soft tissues further deplete the nearby tissues of redundancy and the transferring of healthy tissue from areas of excess becomes necessary ${ }^{[6]}$. Indeed, the complexity of the defects following soft tissue sarcoma resection has increased, as more patients now receive preoperative radiotherapy. Radiation decreases the chance for a successful skin graft, and it also renders the wound edges ischemic. Therefore, well-vascularized tissues are required for reconstruction ${ }^{[7,8]}$.

Reconstruction of soft-tissue defects of the posterior trunk can constitute a challenge for plastic surgeons. Reliable axial pattern flaps for local tissue transfer and recipient vessels for microsurgical reconstruction are scant ${ }^{[9]}$, the wound is often deep and with irregular three-dimensional contour ${ }^{[10]}$. Inadequate amounts of soft tissue can lead to contracture during the healing process, compromising trunk and upper extremity function. Moreover, the exposure of spinous processes may lead to ulceration or pressure sores after soft tissue coverage, so they should be readily removed in order to avoid such complications.

Due to the rich random vascular network multiple local flaps are possible; fasciocutaneous flaps may be raised based on septal and fascial perforators of the axillary subscapular trunks and the posterior intercostals arteries. These flaps provide missing soft tissue for upper-back moderate size defects. Nevertheless, the dorsal trunk hosts several muscles that may be transferred as pedicled flaps such as the latissimus dorsi or the trapezius. Moreover, in selected cases free flaps with vein graft or loops may be used ${ }^{[5-11]}$.

Here we present the case of a patient undergoing bilateral reverse latissimus dorsi myocutaneous (RLDM) flap reconstruction after wide excision of a primary DFSP of the middle back.

\section{CASE REPORT}

A 48-year-old man presented with a painless superficial lesion of the mid-back that had been slowly enlarging for the previous 3 years [Figure 1]. He had no family history of cancer and the past medical history was unremarkable except for hypertension. He had never been a smoker. The clinical examination revealed a large raised lesion, measuring $16 \mathrm{~cm} \times 14 \mathrm{~cm} \times 6 \mathrm{~cm}$ in the middle of the back, extended from the scapula tip to the iliac crest edge (T12-L3). The lesion was barely mobile and hard. A pink placque-like thickening was 


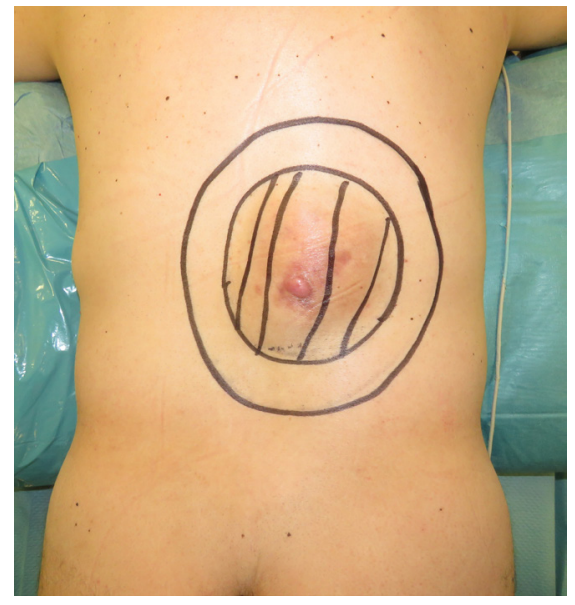

Figure 1. A 48-year-old man with a dermatofibrosarcoma protuberans measuring $16 \mathrm{~cm} \times 14 \mathrm{~cm} \times 6 \mathrm{~cm}$ was present on the mid back
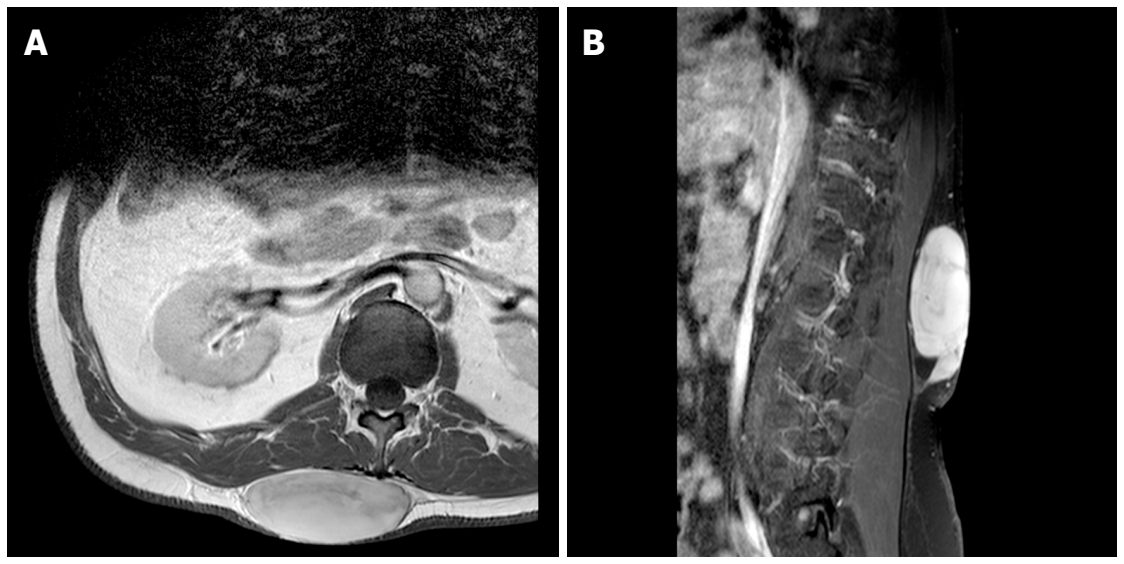

Figure 2. Magnetic resonance imaging showing lesion extent

present in the middle of the lesion. Contrast-enhanced magnetic resonance imaging showed an expansive subcutaneous lesion with no infiltration of deep soft tissues [Figure 2]. The thoracoabdominal computed tomography-scan did not show any other lesion. The patient underwent a percutaneous punch biopsy which revealed a neoplasia composed of CD34-positive spindle cells without atypia or necrosis and with scant mitosis. A diagnosis of DFSP was made.

The flaps were drawn with the patient in a standing position. The resection area measuring $22 \mathrm{~cm} \times 20 \mathrm{~cm}$ was outlined on the back region (lesion: $16 \mathrm{~cm} \times 14 \mathrm{~cm}, 3 \mathrm{~cm}$ oncologically safe distal resection margins). A bilateral RLDM flap was marked, each measuring $24 \mathrm{~cm} \times 10 \mathrm{~cm}$, with its long axis drawn horizontally.

During surgery, the patient was placed in the prone position. First, the tumor was resected with wide margins [Figure 3], encompassing the fascia and superficial layer of the corresponding underlying muscles: the middle and lower part of the right and left latissimus dorsi muscle, and part of the sacrospinalis muscles bilaterally were partially resected. Also the apex of T10-L2 spinous processes were removed. After the excision, a full-thickness defect measuring $22 \mathrm{~cm} \times 20 \mathrm{~cm}$ remained in the middle-lumbar back region [Figure 4]. The size of the two RLDM flaps were reassessed according to the size and shape of the defect. The musculocutaneous flaps, measuring $24 \mathrm{~cm} \times 10 \mathrm{~cm}$, were designed on the superolateral part of the back, between T8-T11 in both sides. The skin and subcutaneous tissue were cut down to the latissimus dorsi 

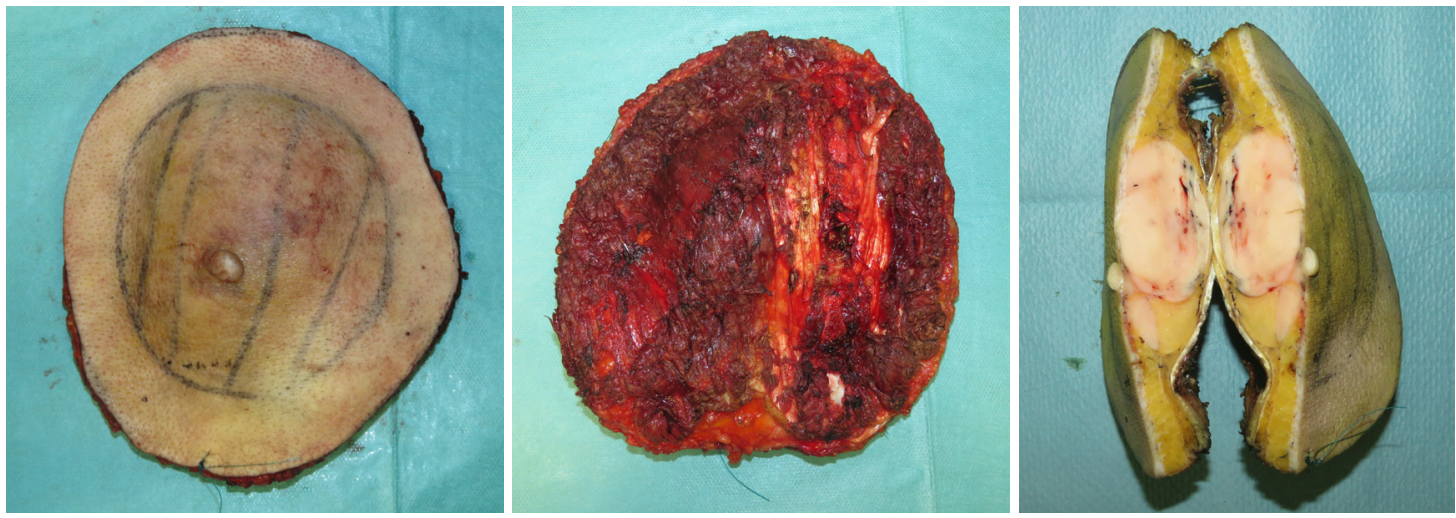

Figure 3. The tumor was widely resected. Intraoperative specimen

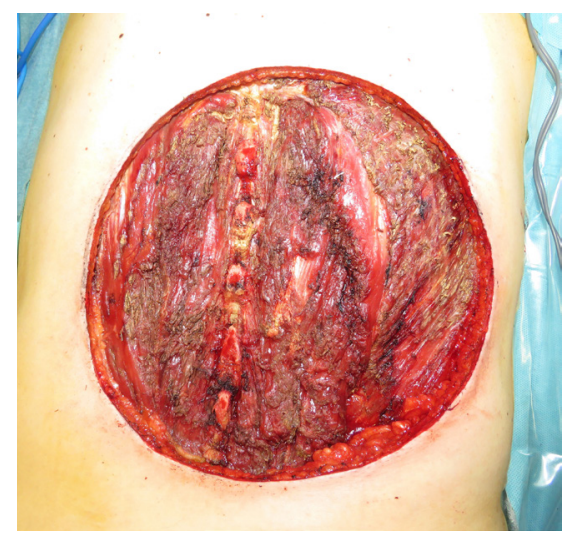

Figure 4. Wound defect after wide local excision of dermatofibrosarcoma protuberans that measured $22 \mathrm{~cm} \times 20 \mathrm{~cm}$ in diameter

muscle, and the flaps were harvested between the latissimus dorsi and the serratus anterior muscles. The thoracodorsal neurovascular bundle was identified, ligated and divided with preservation of the vessels and nerve distributing to the serratus anterior muscle. The myocutaneous flaps were freed from the underlying chest wall along the superior and lower borders of the muscle, preserving dorsal intercostal perforating vessels. When the harvesting was close to the paraspinous region, multiple paraspinal perforators of the posterior intercostal arteries could be seen entering the deep surface of the muscle. We based the two flaps on the 9th and 10th intercostals perforators, in order to allow flap transfer without restriction. The two flaps were transposed to the defect and sutured together [Figures 5 and 6]. The donor sites were closed directly. Four drain tubes were placed ( 2 for the flap donor sites and 2 for the lumbar region).

The patient was kept in a prone position in bed to reduce the tension of the flaps for 2 days. Peri-operative antibiotics were given. He was discharged after 7 days. Drain tubes were removed on the 15th day after the operation. The final pathology showed a completely excised DFSP with clear margins all around. No further therapies were needed. Twelve months after surgery the patient has no evidence of disease recurrence [Figure 7]. The range of motion of shoulder joints and arms were not impaired after surgery. The initial flaps bulging gradually resolved.

\section{DISCUSSION}

The thoraco-lumbar superficial tissues are relatively thick, strongly adherent to the underlying layers, and relatively inelastic. Depending on the extent of the defect and adjacent structures involvement, a variety of 


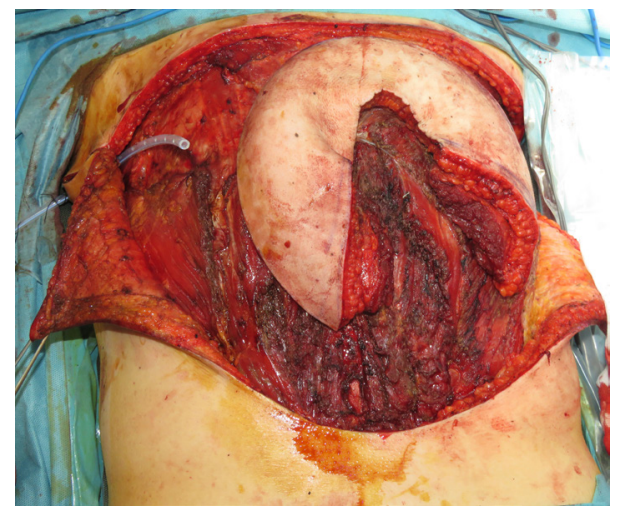

Figure 5. A bilateral reverse myocutaneous latissimus dorsi flap measuring $24 \mathrm{~cm} \times 10 \mathrm{~cm}$ has been raised after tumor resection. Flaps ready to be rotated

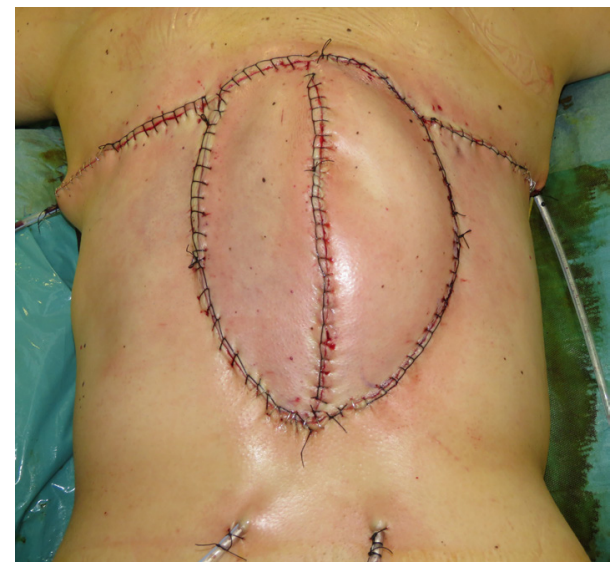

Figure 6. Immediate postoperative result

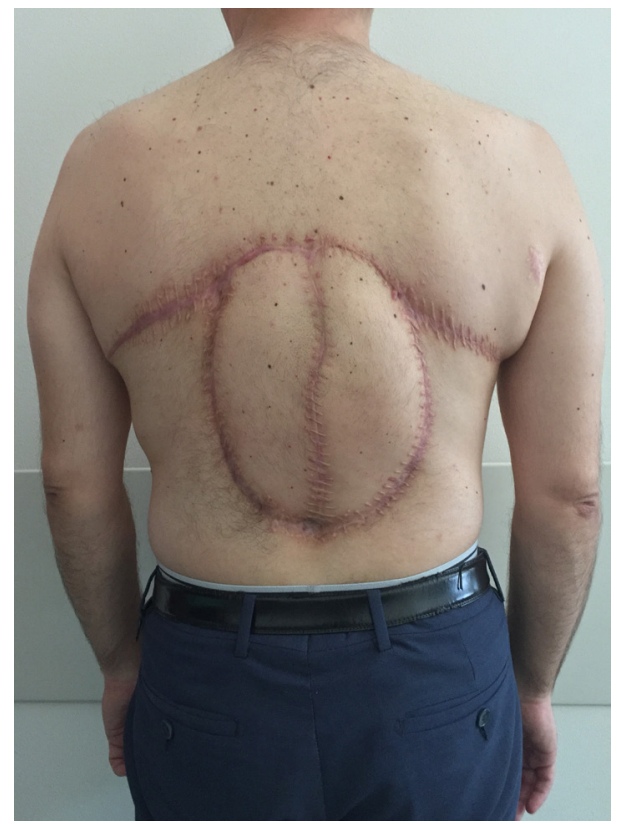

Figure 7. Postoperative picture 12 months after surgery with stable and durable coverage of the wound 
options can be considered for closure of posterior trunk defects. For small wounds that can be closed tensionfree, it may be possible to bring wound edges together for primary closure. This is particularly true when resection is superficial and no vital structures are exposed. Once a large area of tissue has been harvested, however, direct closure becomes more difficult. When underlying vessels, nerves, tendons, ligaments, or even bone have been removed, primary closure is not advisable. Not only it could be impossible to advance flaps far enough to achieve closure, but even if the near tissue might be undermined to allow wound edges to close, the lack of elasticity in the resulting scar tissue is at high risk of causing problems. Furthermore, STS surgery may often result in large composite tissue defects, in which functional structures are frequently exposed and susceptible to infection and mechanical trauma. Therefore, reconstruction of complex defects requires durable and stable coverage of vital neurovascular and bony structures. The posterior trunk softtissue defects can be reconstructed by local skin flaps, fasciocutaneous flaps, perforator flaps, muscle or musculocutaneous flaps and free flaps. Each option has its own advantages and disadvantages.

The abundance of perforating vessels in the posterior trunk offers multiple options for random pattern perforator reconstruction ${ }^{[12]}$; however the use of these flaps is highly dependent on the quality of the surrounding tissue that might be compromised due to radiation and scarring. Compared with myocutaneous flap, there is no muscle composition in perforator flap. Moreover, these flaps are usually thin and not suitable to fill dead spaces. Meanwhile, tedious dissection of the perforators and possible herniation after surgery can sometimes hinder the wide use of perforator flap in lumbar defect reconstruction ${ }^{[13]}$. In these cases the use of muscle or musculocutaneous flaps is advisable.

Myocutaneous flaps are a reliable source of tissue for coverage. The major advantages of myocutaneous flaps are successful wound healing, closure of dead space, and the use of well-vascularized tissue ${ }^{[14]}$.

In the trunk, the pedicled latissimus dorsi flap is usually used for neck, upper back, and thoracic wall reconstructions. The reverse latissimus dorsi flap was indicated for mid/lower back and upper buttock reconstructions.

In 1906, the Italian surgeon Tansini ${ }^{[15]}$ firstly described the utility of the pedicled latissimus dorsi flap for chest wall defects reconstruction after radical breast amputation. The latissimus dorsi muscle flaps offers great variety and options to cover large defects in the mid-thoracic and upper-thoracic posterior trunk. It can be raised up to $30 \mathrm{~cm} \times 40 \mathrm{~cm}$ in size and may be transferred as a muscular (eventually with additional skin grafts) or myocutaneous flap. The latter option makes postoperative monitoring considerably easier. It origins at the thoracical spinous processes, inferior ribs, and iliac crest. The latissimus dorsi muscle inserts at the intertubercular groove of the humerus. Its dominant vascular pedicle is the thoracodorsal artery, which is part of the scapular vascular system, whereas the non-dominant pedicles origin from intercostal and lumbar arteries. It is therefore a class $\mathrm{V}$ muscle according to the popular classification of Mathes and Nahai ${ }^{[16]}$; thus, survival of the flap may also be based on the non-dominant pedicles ${ }^{[17]}$, which would allow utilization of this flap as a "reverse" flap in order to cover contralateral or more caudal defects ${ }^{[9]}$.

The RLDM flap receives its blood supply from the perforating branches of the intercostal and lumbar arteries ${ }^{[17,18]}$. During flap elevation, all medial muscle origin from the spinous processes of the vertebrae can be released, facilitating inferior transposition of the flap. The function of the muscle part in the RLDM flap is the enhancement of bulkiness of the flap in obliterating the dead space. Moreover, multiple perforators of the medial branches of the posterior intercostal vessels and lumbar arteries can be visualized in the midline of the back. Stevenson et al. ${ }^{[17]}$ have demonstrated, that the RLDM flap is mainly nourished by 3 vessels originating from the 9th, 10th, and 11th intercostal arteries and veins located $5 \mathrm{~cm}$ from the midline of the back. These perforators can be preserved if they do not limitate flap transfer, otherwise they can also be dissected. Meanwhile, Maruyama and Iwahira ${ }^{[19]}$ reported that the dorsal perforating pedicle from the 9th 
intercostal vessels can be divided so as to obtain sufficient pedicle length and found that flap survival was not altered. If the flap vascularity is found to be adequate, then perforators can be divided, facilitating further caudal and medial transposition of the flap. During the tumor resection, the surgical oncologist should always be reminded to preserve the integrity of the perforators of the intercostal arteries whenever possible from an oncological point of view.

The bilateral RLDM flap is suitable to cover thoraco-lumbar defect, until L2-L3 but not the sacral region; as matter-of-fact longer flaps could have distal viability and ischemic problems. On the other hand it is not possible to cover defect wider than $25 \mathrm{~cm}$ because larger flaps could have rotational problems.

In our opinion the bilateral RLDM flap is the more reliable surgical option to close a large and deep defect of the mid-low back as in the case described.

\section{DECLARATIONS}

\section{Acknowledgments}

The authors thank Mr. Peter Panton for language editing.

\section{Authors' contributions}

Acted on the demolition surgical step and subsequent patient oncological follow-up, deepened the oncological issue of the paper: Gronchi A, Callegaro D

Played a major role in the reconstructive surgical and scientific field: Bonomi S, Sala L, Cortinovis U

Planned the operation, the writing and the final review of the manuscript: all authors

\section{Financial support and sponsorship}

None.

\section{Conflicts of interest}

There are no conflicts of interest.

\section{Patient consent}

Written informed consent was obtained from the patient for publication of this case report and any accompanying images.

\section{Ethics approval}

Ethical approval was not needed for this case report.

\section{Copyright}

(c) The Author(s) 2018.

\section{REFERENCES}

1. Fletcher CDM, Bridge JA, Hogendoorn P, Mertens F. WHO Classification of Tumours of Soft Tissue and Bone. Fourth Edition. Lyon: International Agency for Research on Cancer; 2013.

2. Thway K, Noujaim J, Jones RL, Fisher C. Dermatofibrosarcoma protuberans: pathology, genetics, and potential therapeutic strategies. Ann Diagn Pathol 2016;25:64-71.

3. Mullen JT. Dermatofibrosarcoma protuberans wide local excision versus mohs micrographic surgery. Surg Oncol Clin N Am 2016;25:827-39.

4. Fiore M, Miceli R, Mussi C, Lo Vullo S, Mariani L, Lozza L, Collini P, Olmi P, Casali PG, Gronchi A. Dermatofibrosarcoma protuberans treated at a single institution: a surgical disease with a high cure rate. $J$ Clin Oncol 2005;23:7669-75.

5. Farma JM, Ammori JB, Zager JS, Marzban SS, Bui MM, Bichakjian CK, Johnson TM, Lowe L, Sabel MS, Wong SL, Douglas Letson G, 
Messina JL, Cimmino VM, Sondak VK. Dermatofibrosarcoma protuberans: how wide should we resect? Ann Surg Oncol 2010;17:2112-8.

6. Zang M, Yu S, Xu L, Zhao Z, Zhu S, Ding Q, Liu Y. Intercostal artery perforator propeller flap for reconstruction of trunk defects following sarcoma resection. J Plast Reconstr Aesth Surg 2015;68:822-9.

7. Langstein HN, Robb GL. Reconstructive approaches in soft tissue sarcoma. Semin Surg Oncol 1999;17:52-65.

8. Chang DW, Robb GL. Recent advances in reconstructive surgery for soft-tissue sarcomas. Curr Oncol Rep 2000;2:495-501.

9. Behr B, Wagner JM, Wallner C, Harati K, Lehnhardt M, Daigeler A. Reconstructive options for oncologic posterior trunk defects: a review. Front Oncol 2016;6:51.

10. Liu Y, Yu S, Song B, Yang L, Zhu S, Jin J. Reconstruction of posterior lumbar defects in oncologic patients using two island flaps of the back in series. Ann Plast Surg 2010;65:326-9.

11. Behnam AB, Chen CM, Pusic AL, Mehrara BJ, Disa JJ, Athanasian EA, Cordeiro PG. The pedicled latissimus dorsi flap for shoulder reconstruction after sarcoma resection. Ann Surg Onc 2007;14:1591-5.

12. Roche NA, Van Landuyt K, Blondeel PN, Matton G, Monstrey SJ. The use of pedicled perforator flaps for reconstruction of lumbosacral defects. Ann Plast Surg 2000;45:7-14.

13. Wong MC, Allison K, Yap LH, Peart F. The pedicled intercostal neurovascular island skin flap for lumbosacral trunk reconstruction. $B r$ J Plast Surg 2004;57:520-4.

14. Misra A, Mistry N, Grimer R, Peart F. The management of soft tissue sarcoma. J Plast Reconstr Aesthet Surg 2009;62:161-74.

15. Tansini I. Sopra Il mio nuovo processo di amputazione della mammella. Gazz Med Ital 1906;12:757. (in Italian)

16. Mathes SJ, Nahai F. Classification of the vascular anatomy of muscles: experimental and clinical correlation. Plast Reconstr Surg 1981;67:177-87.

17. Stevenson TR, Rohrich RJ, Pollock RA, Dingman RO, Bostwick J 3rd. More experence with the "reverse" lastissimus dorsi muscolocutaneous flap: precise location of blood supply. Plast Reconstr Surg 1984;74:237-43.

18. Bostwick J 3rd, Scheflan M, Nahai F, Jurkiewicz MJ. The "reverse" latissimus dorsi muscle and musculocutaneous flap: anatomical and clinical conosiderations. Plast Reconstr Surg 1980;65:395-9.

19. Maruyama Y, Iwahira Y. Latissimus dorsi muscolocutaneous flap: correction of donor-site defect with reverse latissimus flap. Plast Reconstr Surg 1987;80:848-51. 